\title{
Sediment Transport due to Current-Supported Turbidity Currents over an Erodible Bed
}

\author{
Celalettin Emre Ozdemir, Louisiana State University, cozdemir@lsu.edu
}

Sahar Haddadian, Louisiana State University, shadda7@Isu.edu

\section{INTRODUCTION}

Wave- and current-supported turbidity currents (WCSTCs), are one of the chief participants in shaping the marine geomorphology. What makes WCSTCs different from other turbidity currents is that boundary layer turbulence is required to suspend the sediments rather than the self-motion of the turbidity currents. In the presence of a mild slope, the gravitational acceleration drives the suspended sediments offshore (Sternberg et al., 1996; Wright et al., 2001). Depending on what dominates the boundary layer turbulence (BLT), we further define two major subclasses of WCSTCs: (i) wave-supported (WSTCs), and (ii) current-supported turbidity currents (CSTCs). Although significant advances have been made on the details of WSTCs (Ozdemir et al., 2011; Yu et al., 2014; Cheng et al., 2015), less is known about CSTCs. The objective of present study is to investigate the role of alongshore currents on CSTC dynamics over an erodible bottom boundary. The focus here is to identify the possible role of erosion on CSTC dynamics, and assess the coupling between current-induced BLT and suspended sediments for various bed erodibility parameters, i.e. critical shear stress, erosion coefficient, and settling velocity.

\section{MODEL DESCRIPTION}

To fully understand the fundamental characteristics of CSTCs, a direct numerical simulation (DNS) study is employed for a steady, turbulent, particle laden channel flow with a mild transverse slope (Figure 1). Based on dilute sediment concentration assumption the Boussinesq approximation is adopted, and the governing equations are nondimensionalized by shear velocity, $\breve{u}_{\tau}=\sqrt{\check{\tau}_{w} / \rho}$, and channel depth, $h$, as velocity and length scales, respectively. Here, $\check{\tau}_{w}$ is the wall stress and $\rho$ is the fluid density. The nondimensional continuity, momentum, and sediment convectiondiffusion equations, respectively, read:

$$
\begin{gathered}
\boldsymbol{\nabla} \cdot \mathbf{u}^{f}=0, \\
\frac{\partial \mathbf{u}^{f}}{\partial t}+\mathbf{u}^{f} \cdot \boldsymbol{\nabla} \mathbf{u}^{f}=-\nabla p+\frac{1}{F r^{2}} c \mathbf{e}_{\mathrm{g}}+\frac{1}{R e_{\tau}} \nabla^{2} \mathbf{u}^{f}, \\
\frac{\partial c}{\partial t}+\mathbf{u}^{p} \cdot \boldsymbol{\nabla} c=\frac{1}{R e_{\tau} S_{c}} \nabla^{2} c .
\end{gathered}
$$

Here, $\mathbf{u}^{f}$ is the fluid velocity, $\boldsymbol{\nabla} p$ is the pressure gradient, $\mathrm{Fr}$ is the densimetric Froude number, $c$ is the suspended sediment concentration, $\mathbf{e}_{\mathrm{g}}=$ $(0, \sin \theta,-\cos \theta)$ is the unit vector that points the gravitational acceleration, $R e_{\tau}$ is the Reynolds number, and $S_{c}$ is the Schmidt number, where:

$$
R e_{\tau}=\frac{\breve{u}_{\tau} h}{v}, F r=\frac{\check{u}_{\tau}}{\sqrt{(s-1) g h}}, S_{c}=\frac{v}{D} .
$$

The nondimensional form of particle phase velocity reads as:

$$
\mathbf{u}^{p}=\mathbf{u}^{f}+w_{s} \mathbf{e}_{\mathbf{g}} .
$$

Where $w_{s}$ is the settling velocity of the sediments. The sediment erosion/deposition from/to erodible bed, $E$ and $D$, respectively, are determined by using PartheniadesAriathurai type equations (Sanford and Maa, 2001):

$$
\left.\left(-w_{s} c-\frac{1}{R e_{\tau} S_{c}} \frac{\partial c}{\partial z}\right)\right|_{Z=0}=E-D .
$$

In all simulations the $R e_{\tau}$ and $F r$ are specified as constants and equal to $R e_{\tau}=360, \mathrm{Fr}=4 \times 10^{-3}$. The range of critical shear stress and $w_{S}$ are chosen as $\tau / \tau_{c r}=0.7,0.8,0.9, w_{s}=0.04,0.06,0.08$, and $S_{c}=1$.

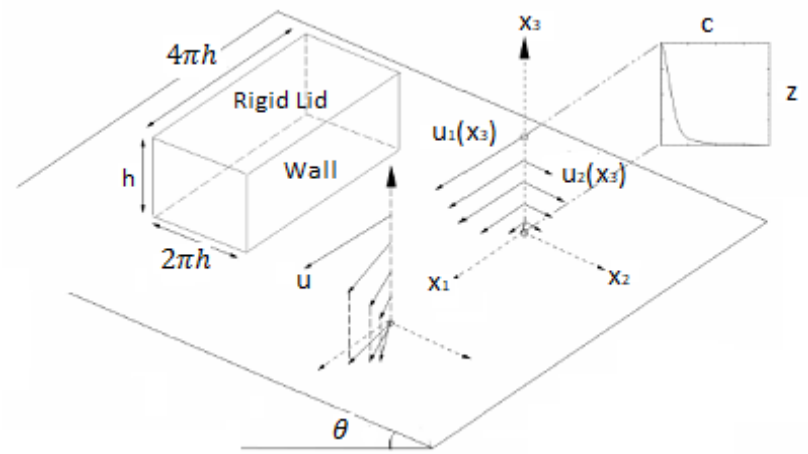

Figure 1- Descriptive sketch of the computational domain. Top and bottom boundaries are specified as rigid lid and wall boundary conditions, respectively. The remaining boundary planes are specified as periodic boundary conditions. The mean current is forced by a uniform pressure gradient in $x_{1}$ direction, and the cross flow in $x_{2}$ direction is driven by density difference created by suspended sediment concentration.

\section{PRELIMINARY RESULTS}

Our preliminary results indicate that there is an inverse relationship between the settling velocity and suspended sediment concentration. We also observed that the production term due to CSTC, nonlinearly varies with the inverse of the settling velocity. With reduction in the settling velocity, turbulence created by CSTC alters the turbulence created by alongshore currents and the CSTC tends to become self-sustaining.

\section{REFERENCES}

Sternberg, R.W., Cacchione, D.A., Paulson, B., Kineke, G.C., Drake, D.E. (1996): Observations of sediment transport on the Amazon subaqueous delta, Continental Shelf Research, 16, 697-715.

Wright, L.D., Friedrichs, C.T., Scully, M. E., (2001): Effects of ambient currents and waves on gravity-driven sediment transport on continental shelves. Mar. Geol. 175, 25.

Ozdemir, C.E., Hsu, T.J., Balachandar, S., (2010): A numerical investigation of fine particle laden flow in oscillatory channel: the role of particle- induced density stratification. J. Fluid. Mech. 665, 1-45.

Ozdemir, C.E., Hsu, T.J., Balachandar, S., (2011): A numerical investigation of lutocline dynamics and saturation of fine sediment in the oscillatory boundary layer, J. Geophys. Res., 116, C09012.

Cheng, Z., Yu, X., Hsu, T.J., Ozdemir, C. E., Balachandar, S., (2015): On the transport of fine sediment in the wave boundary layer due to resuspension/deposition-A turbulence-resolving numerical investigation, J. Geophys. Res. Oceans, 120, 1918-1936.

Sanford, L.P., and Maa, J. P.Y., (2001): A unified erosion formulation for fine sediments, Mar. Geol., 179(1), 9-23. 